\title{
Resistance Training Using Different Hypoxic Training Strategies: a Basis for Hypertrophy and Muscle Power Development
}

\author{
Belén Feriche* $\mathbb{0}$, Amador García-Ramos, Antonio J. Morales-Artacho and Paulino Padial
}

\begin{abstract}
The possible muscular strength, hypertrophy, and muscle power benefits of resistance training under environmental conditions of hypoxia are currently being investigated.

Nowadays, resistance training in hypoxia constitutes a promising new training strategy for strength and muscle gains. The main mechanisms responsible for these effects seem to be related to increased metabolite accumulation due to hypoxia. However, no data are reported in the literature to describe and compare the efficacy of the different hypertrophic resistance training strategies in hypoxia.

Moreover, improvements in sprinting, jumping, or throwing performance have also been described at terrestrial altitude, encouraging research into the speed of explosive movements at altitude. It has been suggested that the reduction in the aerodynamic resistance and/or the increase in the anaerobic metabolism at higher altitudes can influence the metabolic cost, increase the take-off velocities, or improve the motor unit recruitment patterns, which may explain these improvements. Despite these findings, the applicability of altitude conditions in improving muscle power by resistance training remains to be clarified.

This review examines current knowledge regarding resistance training in different types of hypoxia, focusing on strategies designed to improve muscle hypertrophy as well as power for explosive movements.
\end{abstract}

\section{Key Points}

- Despite the fact that the balance of results tends to favor resistance training in hypoxia, no consistent differences in results have been detected between hypertrophy/strength resistance training in normoxia and hypoxia. Moderate resistance loads and moderate altitudes may promote the most favorable physiological and functional changes.

- Ascent to altitude, as opposed to simulated hypoxia, leads to velocity and power improvements that could have positive applications in improving velocity and technical skills in power-related sports. The mechanisms that promote the benefit of this type of hypoxia over normobaric hypoxia still require clarification.

\footnotetext{
* Correspondence: mbelen@ugr.es

Department of Physical Education and Sport, Faculty of Sport Sciences, University of Granada, Crta Alfacar sn, 18011 Granada, Spain
}

- Further research is needed to clarify the efficacy of resistance training specifically oriented to muscle power or hypertrophic gains. Additional research should involve trained athletes and take into consideration nutrition, hydration, and the adjustment of the training load at terrestrial altitude, before proposing new strategies for hypertrophy.

\section{Introduction}

Altitude training is frequently part of an elite athlete's exercise program. By inducing tissue hypoxia due to a lower arterial partial pressure of oxygen $\left(\mathrm{PO}_{2}\right)$, altitude training causes a physiological response that affects performance. Traditionally, the ascent to a higher altitude has been associated with impaired endurance performance $[1,2]$. However, when remaining at altitude, changes in the body systems involved in aerobic energy supply seem to elicit beneficial chronic adaptations improving performance $[3,4]$. Conversely, non-oxidative metabolism-dependent, short-lasting activities (under 
$1 \mathrm{~min})$ seem to offer immediate benefits when performed in altitude conditions $[1,5]$.

A hypoxic environment appears to create advantageous responses in the development of muscle performance with increased hypertrophy and gains in both muscle strength and speed of explosive movements. Despite the importance of resistance training to performance, the muscle response under conditions of hypoxia has not been studied in detail. Very few studies have evaluated the effect of induced hypoxia on anaerobic and aerobic metabolism or the capacity for recovery from different work/rest ratios in repeated exercise training $[6,7]$, as used during resistance training. The combination of the load, sets, repetition of sets, rest, and speed of movement are key factors in understanding the physical and functional muscle changes derived from specific resistance training, as well as the influence that "hypoxic conditions" could have on the results. For hypertrophy and strength gains, when looking for increased muscular cell swelling and metabolite accumulation, traditional methodology usually combines $6-12$ sets of $8-12$ repetitions at low velocity with loads of $65-80 \%$ of 1 repetition maximum (1RM) and 1-3 min of rest in between sets $[8,9]$. For muscle power resistance training, geared to a neuromuscular goal and avoiding metabolic fatigue, sessions usually combine 4-6 sets of 4-6 repetitions with loads of $0-50 \% 1 R M$ for ballistic exercises [10] and 35 min of rest (sometimes this method includes interrepetition rests of 10-60 s, cluster training).

Given different both resistance training purposes, and considering the limited number of studies that have examined muscular adaptation and performance under hypoxic conditions, it is necessary to analyze the differences in the experimental designs, participant training levels, and the type and severity of hypoxia before drawing conclusions. In the following section, muscle hypertrophy and power trainability under hypoxic conditions will be reviewed in order to clarify the consistency of the results available.

Throughout the different sections of this review, different types of hypoxia and hypoxic training strategies will be referred to. Current training trends in hypoxia combine different types and dosages of hypoxia $(\mathrm{H})$ resulting in numerous possible combinations $[11,12]$ :

- Hypobaric hypoxia (HH), or altitude, produces the hypoxic effect (decrease the availability of oxygen in the tissues) by reducing the barometric pressure, mainly by ascent to altitude or by using hypobaric chambers. The reduction in barometric pressure reduces air resistance to the movement as a result of lower air density. An ascent to altitude also results in reduced air temperature and humidity.

- Normobaric or systemic hypoxia (NH) provides the hypoxic effect by reducing the oxygen pressure in the inspired air (nitrogen dilution or oxygen filtration).
In this sense, natural or artificial methods of hypoxia can be used for training, resulting in strategies such as live high-train high (LHTH), live high-train low (LHTL), or live low-train high (LLTH) among others. Intermittent hypoxic devices are also used during training sessions (IHT) or resting periods. Training at low altitude allows the athlete to maximize performance by maintenance of sea-level training intensity and oxygen flux [11]. Extensive literature relating to the use of these combinations in endurance training exists, although its application in resistance training is mainly limited to the use of intermittent artificial hypoxic exposure while resting under normoxic conditions (LLTH) to increase hypertrophy, also called intermittent hypoxic resistance training (IHRT) [13].

\section{A Description of the Mechanisms and Metabolic Factors Related to the Hypertrophic and Functional Muscle Response in Acute and Chronic Hypoxic Conditions}

Several studies have tested the degree to which hypoxic conditions are able to produce beneficial changes to muscle strength and hypertrophy [14-17]. These studies follow on from previous research into the apparent beneficial effects on muscle hypertrophy and strength gains of low resistance training (20-50\% 1RM) when combined with blood flow restriction (BFR) in different subject populations [18-24]. The main mechanisms proposed for these improvements relate to responses to the metabolite buildup [25-27] (Fig. 1). Then, moderate intensity resistance training under hypoxic conditions enhances exerciseinduced metabolic stress mechanisms (anabolic hormones, cytokines, reactive oxygen species, and oxidative stress factor, among others), which clearly have an important role in muscle growth [17, 27-30]). Accordingly, several studies have been conducted in hypoxia aiming to achieve strength and hypertrophic benefits [14, 17, 21, 22, 30, 31].

On the other hand, improvements in sprinting, throwing, and jumping at altitude have been reported [1, 2, 5, 32]. To date, researchers have mainly used the hypothesis of reduced energy costs to account for improved isolated high-speed actions [33] through a reduction in aerodynamic resistance in approximate proportion to the square of the velocity (e.g., when cycling, running, or throwing objects) $[1,5,32]$. However, although the underlying mechanisms are not completely defined, modified motor unit recruitment patterns due to increased anaerobic metabolism release $[34,35]$ could also partly explain these improvements. Furthermore, it could be considered that if this occurs in isolated movements, some benefit could also be obtained during a prolonged period of specific explosive resistance training at altitude, opening up a new line of investigation that considers the effects of both air composition and its resistance. 
METABOLIC FACTORS INVOLVED IN MUSCLE HYPERTROPHY RESPONSE
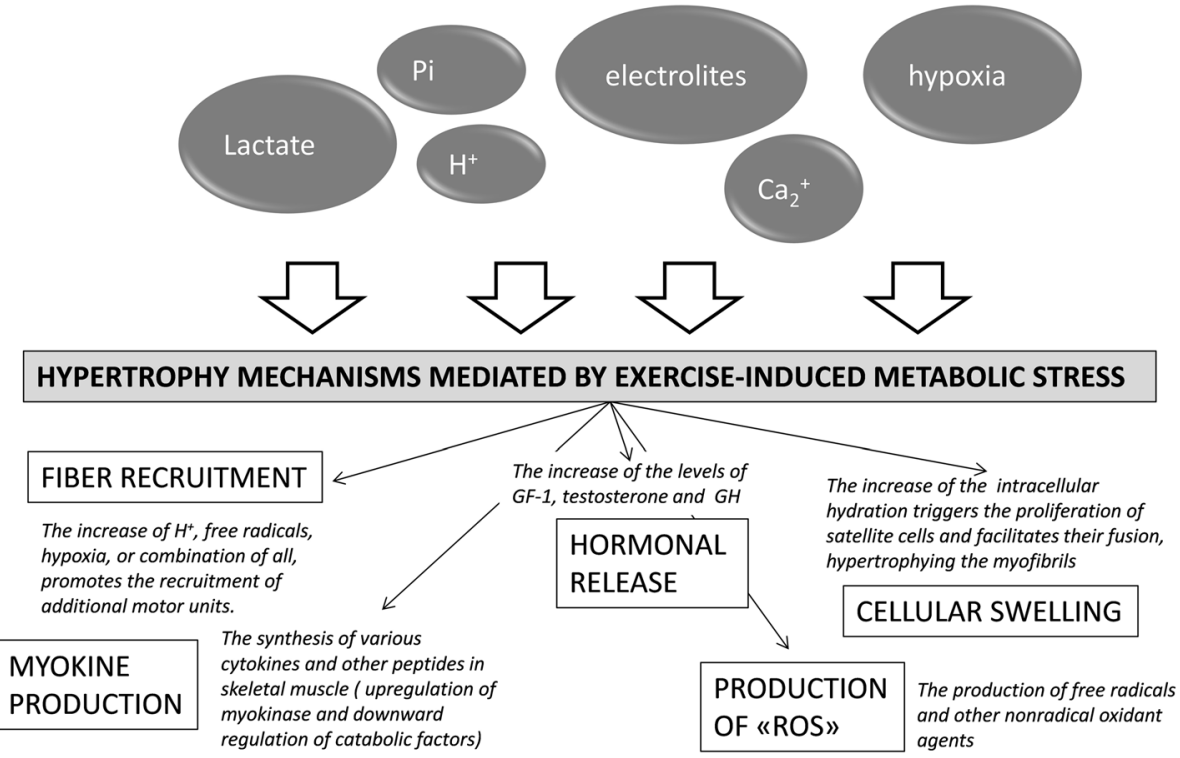

Fig. 1 Hypertrophy mechanisms mediated by metabolites

Up until now, the impact of whatever type of hypoxia on muscle performance and the biomechanics of specific movements in sport has not been examined in depth. Alterations in the biomechanical and neuromuscular components associated with force production are some of the factors recently suggested by Chapman et al. [36] that influence changes in performance following altitude training. According to this, the improvement in speed movement can also be attributed to an enhanced firing frequency of motoneurons and spinal reflexes. Acute simulated hypoxia has been related to the increase of the spinal excitability [37]. Additionally, Tomazin et al. [38] recently observed a greater increase in the H-reflex amplitude of the soleus muscle at a terrestrial altitude of $2320 \mathrm{~m}$ when compared to that at sea level $(\sim 35 \% ; p<0.05)$, a response that could be linked to a direct effect of hypoxemia on the supraspinal structures. There may, however, be further contributory mechanisms, such as increased anaerobic metabolism [27, 34, 35] and/or reduced air density, as mentioned above [33], that influence muscle contraction properties and thus improve explosive speed [1, 5] (Fig. 2). In fact, breathing hypoxic gas mixtures while training seems to display a tendency for fast fiber areas to increase in size following training compared to breathing normoxic air [39] that could be also linked to a greater fast fiber recruitment. Accordingly, other studies have revealed maximal power gains during a force-velocity $(F-V)$ curve [40-43], as well as 1RM gains [40-42], after acute exposure to real moderate altitude in bench press, back half squat, and squat jump exercises. However, Scott et al. [44] detected no changes in force and power performance during back squat and dead lift exercises (80\% 1RM) at simulated moderate and high altitudes.

\section{Hypertrophy Trainability in Conditions of Hypoxia}

Scott et al. [24] reviewed the hypothetical benefits on muscle strength and muscle size of intermittent hypoxic resistance training compared to BFR. Resistance training under conditions of systemic hypoxia $(\mathrm{NH}$; achieved by nitrogen dilution, oxygen extraction in an environmental chamber or wearing a face mask) could be considered as an alternative to BFR while avoiding problems such as restricting training to only the limbs, pain, petechial hemorrhage, and dizziness related to the use of compression cuffs [24]. As has been mentioned, moderate intensity resistance training under hypoxic conditions enhances the build-up of metabolites, which clearly have an important role in muscle growth [17, 27-30]. However, the literature does not provide data addressing the effect of a resistance training period at terrestrial altitude, and conclusions obtained during IHRT in normobaric hypoxia are not clear. Gains in muscle mass and strength after a hypoxic training period display no consistent differences when compared with those observed in normoxia (Table 1).

The effect of the hypertrophic resistance training at terrestrial altitude on muscle mass has not been studied in detail. Early studies associated the ascent and extended periods spent at high altitude $(>5500 \mathrm{~m})$ with up to a $15 \%$ muscle mass loss [45] and reduced strength gains $(-6.4 \%)$ compared to normoxia [46]. Explanations for this have included altitude-induced protein synthesis 


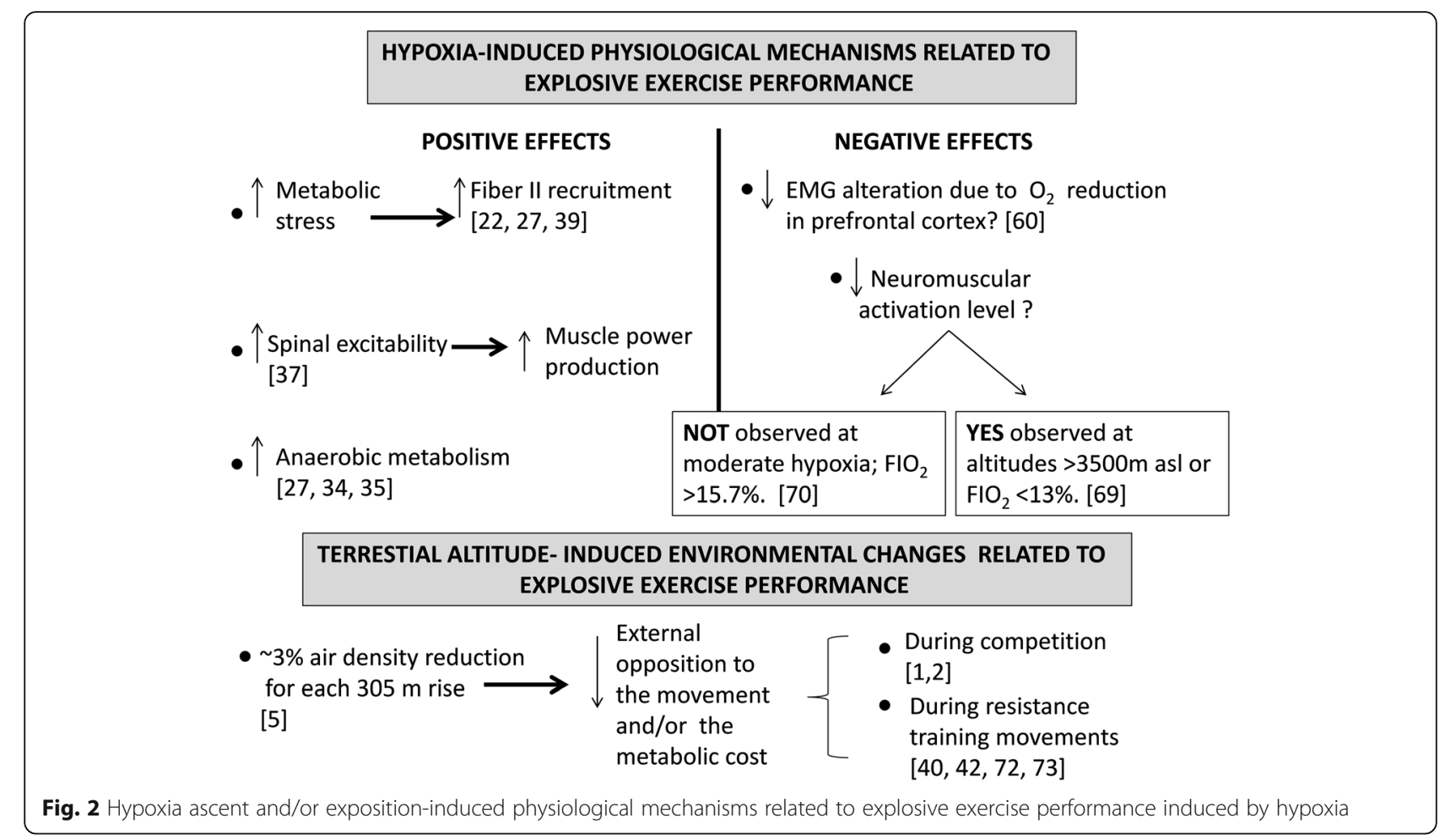

rate reduction $[47,48]$ or increased protein degradation during exercise [49], leading to a negative synthesis/degradation of protein balance. Nevertheless, training camps are usually held at moderate altitudes (1800-2500 m), while no data are reported in scientific literature about hypertrophic resistance training during intermittent or chronic periods at terrestrial altitude; protein metabolism seems to be unaffected by $\mathrm{O}_{2}$ availability at moderate simulated altitudes in acute $\mathrm{NH}$ [50].

\section{Effect of Low-Load Resistance Training on Hypertrophy in Conditions of Hypoxia}

Several interesting studies have been analyzed in order to highlight the effect of low-load resistance training in hypoxia on strength and muscle growth (see Table 1). Additional muscle size gains of $3.2 \%$ were detected by Manimmanakorn et al. [21, 22] after 5 weeks of lowintensity resistance training under intermittent normobaric hypoxia $\left(\mathrm{SaO}_{2}\right.$ of $80 \%, \sim 4000 \mathrm{~m}$ asl $)$ when compared with normoxia (3 sets of the repetitions to failure at $20 \%$ $1 \mathrm{RM}, 30 \mathrm{~s}$ of rest between sets). Traditionally, multiple sets of loads over $65-70 \%$ of 1 RM were considered necessary for significant hypertrophy [8]. However, current trends suggest that low-load resistance training is also able to induce muscular hypertrophy through mechanisms not related to mechanical stress. In this case, muscle growth is highly dependent on metabolic factors, and training sets should be conducted to failure [27]. These two points must be considered in both normoxic and hypoxic conditions despite the fact that there is an accelerated build-up of metabolites observed in hypoxia during resistance training with moderate loads [17, 27-30], but not with low loads [51]. For instance, similar lactate and anabolic hormone responses were observed for squat exercises ( 5 sets of 15 repetitions at 30\% 1RM, $90 \mathrm{~s}$ of rest) in conditions of normoxia, as those for systemic hypoxia (15\% of the inspired oxygen fraction $\left[\mathrm{FiO}_{2}\right]$ ) [51]. For this reason, performing a high number of repetitions in each training set is an important detail that could explain the positive results of Manimmanakorn et al. [21, 22] given the absence of strength and hypertrophic changes in other studies under similar conditions [14]. Manimmanakorn et al. [21, 22] compared three experimental situations: normoxia, normobaric hypoxia, and BFR, and participants were instructed to match the repetitions performed by the BFR group to ensure equal training loads between groups ( $\sim 28$ and 36 repetitions in knee flexion and extension respectively). However, Friedmann et al. [14], when comparing the effect of 4 weeks of resistance training in normoxia and normobaric hypoxia $\left(\mathrm{FiO}_{2}=12 \% ; \sim 4500 \mathrm{~m}\right.$ asl), used a fixed number of sets of repetitions in the same exercises (6 sets of 25 repetitions at 30\% 1RM, $60 \mathrm{~s}$ of rest between sets). Additionally, the 30-s difference in rest periods between these studies could also have had a favorable influence on the Manimmanakorn et al. exercise routine as shorter recovery periods may heighten the metabolic stimulus to enhance the anabolic response [13]. Conversely, in these three studies [14, 21, 22], endurance- 


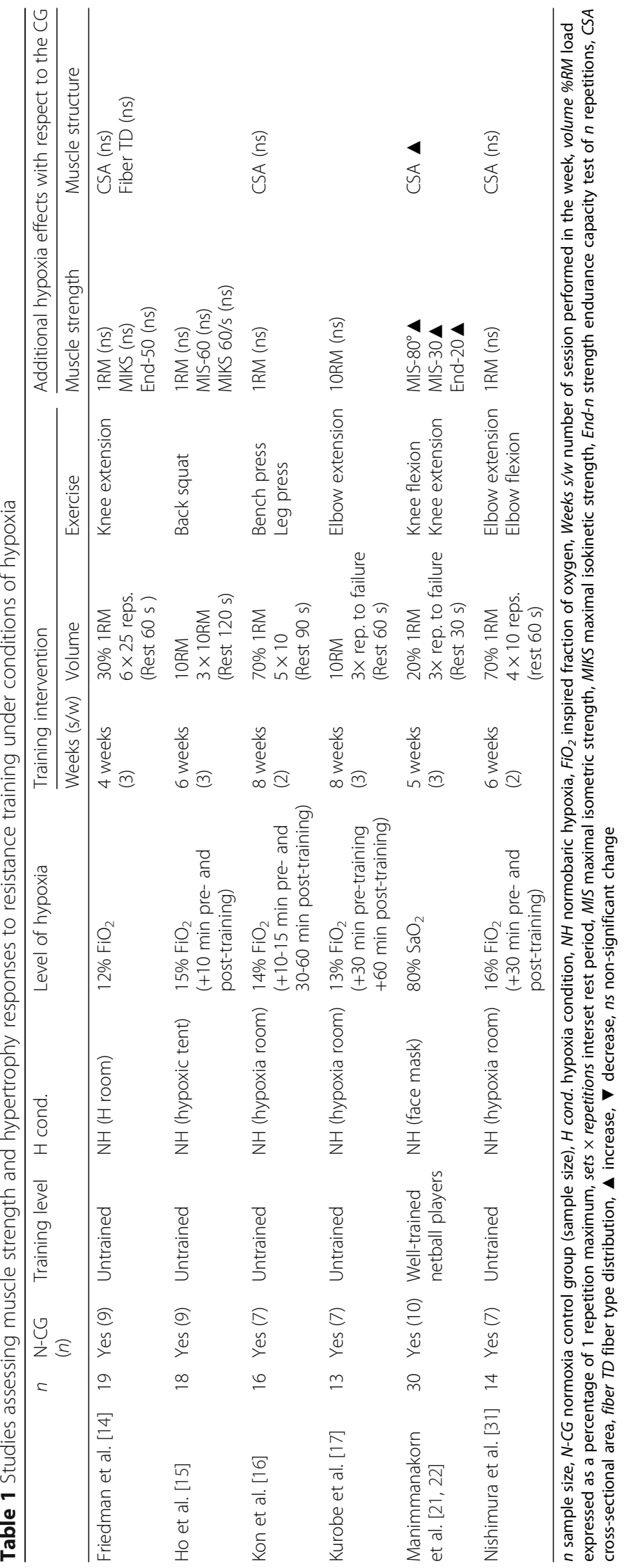


force was improved in hypoxia despite the exposure to lower $\mathrm{SaO}_{2}$ than in hypoxic dose recommendations $\left(<3500 \mathrm{~m}\right.$ or $\left.\mathrm{SaO}_{2}>80 \%\right)$ [50], probably due to improved metabolic efficiency [22].

\section{The Effect of Moderate-Load Resistance Training on Hypertrophy in Conditions of Hypoxia}

When analyzing research conducted by using moderate resistance training programs $(65-80 \% 1 \mathrm{RM})$, three of the four available studies (see Table 1) display results which do not reveal a clear effect of systemic hypoxia on muscle strength and hypertrophy with respect to the same training in normoxic conditions. Accordingly, the influence of acute exercise-induced endocrine responses in muscle growth has recently been questioned, and may not have the expected anabolic effects in healthy subjects [52]. This contrasts with the evidence described in other studies in which a hypertrophic resistance training program produced strength gains [31], and muscle cross-sectional area (CSA) increases driven by the growth hormone response [26].

Compared to normoxic conditions, Nishimura et al. [31] observed that 6 weeks of resistance training (4 sets $\times 10$ repetitions at $70 \% 1 \mathrm{RM} ; 1$ min rest) under normobaric hypoxic conditions $\left(16 \% \mathrm{FiO}_{2}\right)$ improved arm strength levels (66 vs $48 \%$, non-significant), and a change in CSA in hypoxia was shown at early stage throughout the training period (1.3-1.9\% increase in CSA) in untrained subjects. Similar results were reported by Kurobe et al. [17], who associated the better results of 8 weeks of resistance training of the arms ( 3 sets of $101 \mathrm{RM}$; 1 min of rest) while breathing a reduced $\mathrm{O}_{2}$ air (13\% $\mathrm{FiO}_{2}$ ) with higher growth hormone secretion. This is consistent with the greater blood lactate and anabolic hormone responses observed by Kon et al. [28] after a bench press and leg press resistance training session (5 sets of 14 repetitions at 50\% $1 \mathrm{RM} ; 1$ min rest) in $\mathrm{NH}$ conditions of $13 \%$ of $\mathrm{FiO}_{2}$ despite the fact that the load used was just below the low-limit threshold of what is considered a "moderate-load" (but nevertheless above what is considered a lower load). No differences between conditions in the theoretical maximal strength were reported by Kurobe's team, but 1RM estimates in high repetition tests to failure (10RM, $75 \% 1 \mathrm{RM}$ ) may be unreliable due to fatigue and mechanical stress [53, 54]. Contrary to these findings, Ho et al. [15] concluded that 6 weeks of short-term resistance training (3 sets of 10RM; 2 min rest) under $\mathrm{NH}\left(15 \% \mathrm{FiO}_{2}\right)$ offered no additional benefit to muscular performance or body composition. Likewise, Kon et al. [16] observed no strength or muscle size gains in response to bench press and leg press ( 16 sessions of 5 sets $\times 10$ repetitions at $70 \% 1$ RM; $90 \mathrm{~s}$ of rest) though they did detect enhanced skeletal muscle endurance and angiogenesis.

\section{Inconsistencies Among Studies of Hypertrophic}

\section{Resistance Training Effects Mediated by Hypoxia}

The lack of consensus among reported studies may be attributed to differences in protocols such as training intensity (light or moderate loads), number of sets (3 to 6), rest period between sets (30 to $120 \mathrm{~s}$ ), muscles worked (arms, legs, chest), training program duration (4 to 8 weeks), and severity of hypoxia (from 12 to $16 \% \mathrm{FiO}_{2}$ ). Additionally, all studies were performed in untrained subjects and the muscle stimulus employed was lower than recommended for hypertrophy $[8,55]$. In untrained subjects, neural modifications start during the early stages of training $[14,56]$ with an individual long-phase that could, in part, explain the different strength and/or muscle size gains observed among studies.

In the reviewed studies, hypoxia levels were performed at simulated hypoxia which ranged from 12 to $16 \% \mathrm{FiO}_{2}$, which although being at or slightly above the limit $\left(\sim 12 \% \mathrm{FiO}_{2}\right)$ is not likely to produce discrepancies. During $3.5 \mathrm{~h}$ following moderate-intensity resistance training (6 sets of 8 repetitions at 70\% 1RM) under acute severe hypoxia ( $4300 \mathrm{~m}$ asl, $\left.12 \% \mathrm{FiO}_{2}\right)$, a relationship exists between protein synthesis rate and arterial oxygen saturation $\left(\mathrm{SaO}_{2}\right)\left(r^{2}=0.49, p=0.04\right)$. This means that for $\mathrm{SaO}_{2}<80 \%$ or altitudes $>3500 \mathrm{~m}$, hypoxia may delay the anabolic response to resistance training compared to normoxia, although after $3.5 \mathrm{~h}$, responses should be comparable [50]. No data concerning the effect of hypertrophic resistance training at terrestrial altitude are available, so differences between simulated and terrestrial hypoxia cannot be reported. At high or moderate altitude, other factors could also promote muscular changes over longer periods, such as the following: (1) reduced food intake (10-50\%) linked to a loss of appetite [57] and/or change in diet; (2) increased energy expenditure due to a higher basal metabolic rate [58] and/or physical activity not matching energy intake; (3) dehydration [59]; and (4) absence of load adjustment during hypertrophic resistance training at altitude. Indeed, the reduction of the training load stimulus to the muscle during altitude training can be considered as one of the mediating factors related to the loss of muscle mass traditionally linked to an altitude camp. This can occur when the training load used in normoxia is maintained during the altitude stage. Therefore, if the 1RM absolute load improves by $\mathrm{HH}$ and the resistance training load is not adjusted accordingly, the stimulus to the muscle during the resistance altitude training will be reduced. In this context, Feriche et al. [40] describe a concentric bench press 1RM improvement at acute terrestrial altitude $(\sim 5.6 \% ; \mathrm{ES}=1.1)$ with respect to the change observed in normoxia and NH. Similar results have also been described in the half squat [41]. However, there are no data from any longitudinal study at terrestrial altitude. 
Finally, another point of consideration is that the minimum time needed to detect significant hypertrophic muscular changes in athletes is around 8 weeks [9]. The research revised deals with study periods ranging from 4 to 8 weeks, which could contribute to the discrepancies observed among the results, as well as limiting the use of terrestrial altitude. Changes in muscle size and strength resulting from resistance training during an extended period at real moderate altitude still require clarification. Moreover, real moderate altitude training camps normally last 3 weeks, which is an insufficient time to achieve the target, although the hypertrophic training must be carefully adapted to avoid the undesired results previously mentioned. For this reason, when pursuing hypertrophy, longer hypoxic training programs which simulated normobaric hypoxia is generally selected, usually involving IHRT sessions.

According to Scott et al. [13], more control research is needed to evaluate the real influence that hypoxia constitutes in encouraging hypertrophy and strength gains. The potential effect of hypoxia on growing muscle mass, specially by the marked metabolic stress that the exercise performed under hypoxic conditions causes, is well documented [27, 28, 30] although questioned in some contexts by the lack of positive results [14-16]. This information should be considered in future investigations conducted in hypoxia and take into account previous conclusions about how to apply hypertrophic methods $[8,9]$, type and severity of the hypoxia $[11,12,50]$, influence of the training level on the sample [14,56], and recommended length of the intervention [9]. Moreover, the ideal strategies for resistance training during altitude camps and other types of interventions combining terrestrial altitudes should also be explored.

\section{Muscle Power Trainability in Conditions of Hypoxia}

The influence of altitude or hypoxic conditions on muscular function during muscle power resistance exercises has not been examined in detail. Exercise-induced fatigue may have a central element which may or may not include a peripheral cause. Although the direct impact of hypoxia on the brain cannot be ruled out [60], sensory feedback of metabolite accumulation due to lower $\mathrm{O}_{2}$ availability (such as $\mathrm{H}^{+}$or $\mathrm{Pi}$ ) may explain why the central command and power output in hypoxia is reduced [61]. Literature describes a direct but moderate influence of the inspired $\mathrm{O}_{2}$ fraction on the central nervous system. This conclusion was reached by Millet et al. [62] after studying the response of intermittent isometric unilateral knee extensions to failure with and without blood flow restriction (BFR, via a cuff), in $\mathrm{N}$ and while breathing a reduced $\mathrm{O}_{2}$ air $(\mathrm{NH}$ of $11 \% \mathrm{FiO}_{2}$ and $84 \% \mathrm{SaO}_{2}$ ). Both, hypoxia and the occlusion cuff, affected the number of repetitions. However, considering the muscle similarly affected in the two conditions, performance was slightly but significantly lower during $\mathrm{NH}$ than in $\mathrm{N}$ with cuff on. The design used in this study leads us to conclude that systemic hypoxia has a direct influence on the central drive, independent of the factors developed within the working muscles. During severe levels of hypoxia, the type of muscle contraction or the total muscle mass involved in exercise can also limit the influence of this mechanism [62].

Perrey and Rupp [63] reviewed studies analyzing the effects of acute or prolonged exposure to terrestrial high altitude $(\geq 3700 \mathrm{~m})$ on the contractile properties of the muscle. Under conditions of acute altitude, muscle function was altered after intermittent contractions, while modifications to chronic hypoxia seemed to minimize the effect on skeletal muscle function. Impaired muscle function in response to high altitude was especially appreciable during exercise protocols involving prolonged isometric muscle contractions $(<30 \%$ of the maximal voluntary contraction), and during repeated submaximal intermittent contractions, both of which depend principally on systemic $\mathrm{O}_{2}$ transport [63]. Besides muscle deterioration due to high altitude, reduced muscular power has also been described [64].

In contrast, terrestrial moderate altitude throws up different results. Two previous studies reviewed the effect of altitude on elite athletes' performance in sprints from 100 to $400 \mathrm{~m}[1,2]$, and throwing and jumping performance [1]. As was predicted by mathematical models [33], enhanced sprint performances $(0.2-0.7 \%)$, the hammer throw and triple and long jump $(\sim 1 \%)$ are described at altitudes above $1500 \mathrm{~m}$ [1]. Nowadays, there is no doubt about the benefit of terrestrial altitude on these athletic disciplines. Nevertheless, some authors suspect that the slower takeoff speeds in high jump, or the slower velocity in the hurdle race (i.e., from 10.3 to $8.5 \mathrm{~m} \mathrm{~s}^{-1}$ in $100 \mathrm{~m}$ sprint and 110 hurdles, respectively, in men), reduces the influence of aerodynamic drag and it is this that accounts for the minimal effect on performance between altitudes [1]. However, despite the greater difference in speed compared with sprints, jumps, and throws, the changes described in muscle power and velocity during isolated resistance exercises (back squat, bench press or squat jump) from the first hours following ascent [40-43] indicate that some kind of relationship between $\mathrm{HH}$ and muscular function must exist, independently of the changes in speed linked to air density (Tables 2 and 3).

Very frequently, power and velocity assessed during the $F-V$ curves are measured by means of linear transducers attached to the bar, making it difficult to identify if athletes applied more force at altitude or if the results are produced by lower resistance to movement. To analyze this, García-Ramos et al. [43] examined the leg extensor muscle response using a $F-V$ curve and unloaded jumps 


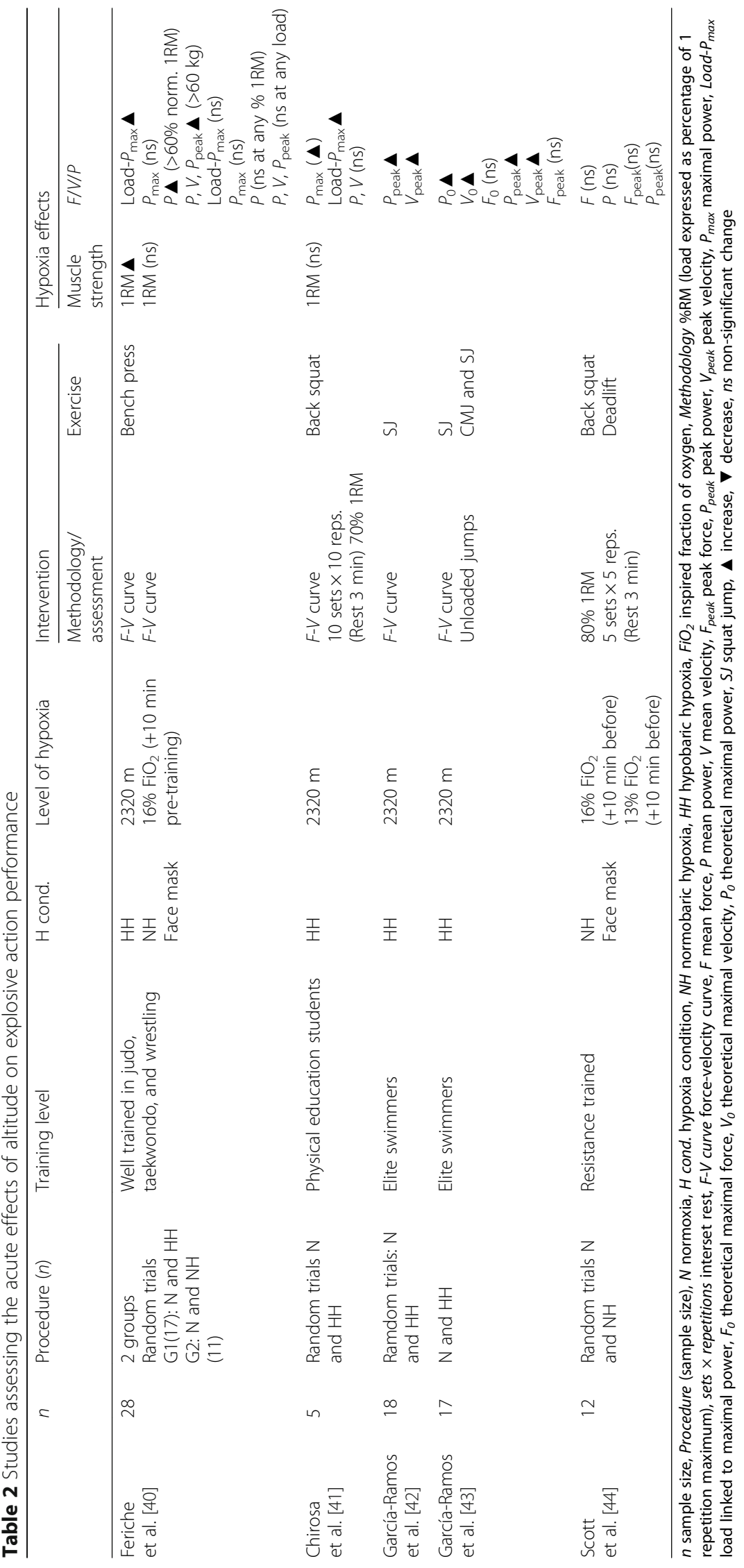




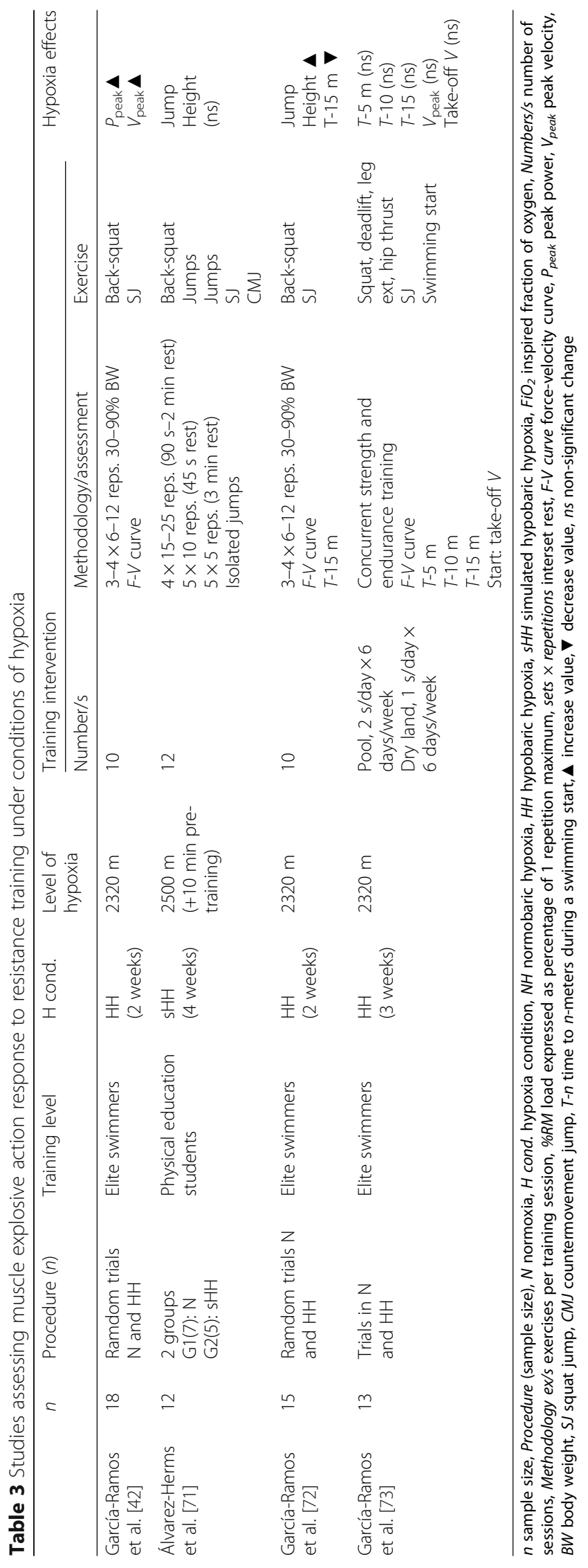


(CMJ and SJ) in 17 elite swimmers before and just after an ascent to terrestrial moderate altitude. The novel aspect of this study is that during the $F-V$ curve, the maximum values of force and velocity at each load were recorded by a force platform and a linear velocity transducer respectively. Thus, these variables were modeled by a linear regression $\left[F(V)=F_{0} a V\right]$, where $F_{0}$ (force intercept at zero $V), V_{0}$ (velocity intercept at zero force), and maximum power output $\left(P_{0}=F_{0} V_{0 / 4}\right)$ were considered as the maximal mechanical capabilities of the neuromuscular system to generate force, velocity, and power, respectively [65]. The results revealed higher magnitudes in $P_{0}(+6.79 \% ; p<0.01)$ of the leg extensors at altitude, which were linked to an increase in the $V_{0}(+7.60 \% ; p<0.05)$, while no changes for the $F_{0}(+0.02 \%)$ were achieved. In addition, the results for unloaded jumps performed on a force platform showed a clear tendency towards improvements in the amount of force applied when performed at altitude, with jump height increasing by an average of $3.4 \%$. These results highlight the influence that the aerodynamic drag forces could have on velocity and show a clear altitude effect on the $F-V$ relationship at the same absolute load [40, 42, 43], sustaining the hypothesis that the hypobaric hypoxia and muscular function relationship must, at some point, converge in addition with the additional benefit produced by the lower aerodynamic resistance on isolated explosive movements.

\section{Terrestrial or Simulated Altitude: Effect on Explosive Muscle Performance}

Despite the reduced $\mathrm{O}_{2}$ content of air during a terrestrial or simulated exposure to hypoxia, differences in barometric pressure can also affect performance in repeated (IHRT) or isolated high-speed explosive actions. In 28 combat sport athletes divided into two homogeneous groups, $F$ - $V$ curves in the bench press were compared between $\mathrm{N}$, terrestrial moderate altitude ( $\mathrm{HH} 2320 \mathrm{~m}$ asl), and normobaric hypoxia $\left(\mathrm{NH} 15.7 \% \mathrm{FiO}_{2}\right)$ [40]. Results indicated a marked effect on the $F-V$ curve of acute $\mathrm{HH}$ compared to negligible effects of $\mathrm{N}$ and $\mathrm{NH}$. Acute $\mathrm{HH}$ led to a $3.2 \%$ mean increase in the load linked to mean maximal power, along with clear improvements in mean power, peak power, and peak velocity for the same absolute load. Hypobaric hypoxia also accounted for a $6 \%$ increase in 1RM after the ascent. This could be considered unsurprising given the confirmed relationship between mean velocity and weight lifted according to percentage $1 R M$ [66-68], and the fact that this velocity is improved at terrestrial altitude $[40,42]$. Using as a reference the $1 \mathrm{RM}$ recorded for $\mathrm{N}$, the mean power curve for $\mathrm{HH}$ was shifted upwards and to the right, indicating that mean power would be overestimated for loads $\geq 60 \%$ of $1 \mathrm{RM}$, compared to the curve obtained using as reference the corresponding $1 \mathrm{RM}$ recorded for $\mathrm{HH}$ [40]. The lack of power output change in $\mathrm{NH}$ concurs with the findings of Scott et al.
[44]. Power and force trends over 5 sets of 5 repetitions at $80 \%$ of $1 \mathrm{RM}$ for acute moderate and high $\mathrm{NH}$ (13 and $16 \% \mathrm{FiO}_{2}$ ) failed to vary from trends recorded in normoxic conditions.

According to other findings, and with evidence of physiological and metabolic responses induced by acute $\mathrm{NH}$ exercise (i.e., cardiovascular and hormonal [17, 28, 30, 44]), velocity, power, and maximum dynamic strength after basic strength exercises show benefits from $\mathrm{HH}$ which are not found in $\mathrm{N}$ or $\mathrm{NH}$ [40-42]. A relationship has also been identified between metabolic stress induced by $\mathrm{H}^{+}$elevation because of low $\mathrm{SaO}_{2}$ and the recruitment of fast twitch muscle fibers [27]. While abnormalities in muscle electromyographic activity have been observed in conditions from acute hypoxia ( $3500 \mathrm{~m}$ asl; $13 \% \mathrm{FiO}_{2} ;$ [69]), moderate altitudes do not lead to these detrimental effects [70]. Additionally, electromyographic activity at $\mathrm{NH}$ has been shown to be similar to that at normoxia during maximal voluntary contractions and power output [22]. For isolated shortburst actions involved in $F$ - $V$ curves $(\sim 5 \mathrm{~s}$ plus $3-5 \mathrm{~min}$ rest), hypoxic benefits to performance were not observed in moderate or high NH (16-13\% FiO2) [40, 44], and only improved at terrestrial altitude [40]. This challenges the idea that the breathing of air impoverished in $\mathrm{O}_{2}$ is solely responsible for inducing a switch from type I to II fibers [22] making the movement faster due the intrinsic capacity that larger motor neurons have to drive the impulses at higher speeds. It is likely that differences between results obtained at $\mathrm{NH}$ and $\mathrm{HH}$ seem mediated by other factors and/or interactions not yet investigated.

Álvarez-Herms et al. [71] did not observe any change in the height achieved during an isolated free squat jump (SJ) and countermovement jump (CMJ) after 4 weeks of endurance resistance training in simulated hypobaric hypoxia of $2500 \mathrm{~m}$ asl ( $n=6$ men and 1 women) and normoxic conditions ( $n=3$ men and 2 woman). This result is not surprising since the training was oriented to endurance. In contrast, a recent study conducted on 18 young male swimmers of a junior national team found mean peak power and peak velocity improvements of $12.1 \pm 1.8 \%$ and $6.6 \pm 1.2 \%$, respectively, for loaded SJ after ascending to a terrestrial moderate altitude [42]. This study also demonstrated the persistence of altitudeinduced improvements in jump performance after 2 weeks of exposure to real moderate hypoxia, showing mean improvements in both variables of 7.8 and $4.4 \%$, respectively. Moreover, significant correlations between the percent change in jump height and the percent change in swimming start performance were also obtained following a short-term training program of 17 days [72]. These three studies show that moderate exposure to real or simulated hypobaric hypoxia does not impair the ability to apply force rapidly [42, 71, 72], and this capacity is likely to improve with specifically target- 
oriented training [42, 72]. In accordance with this hypothesis, it has been recently observed that 3 weeks of training high-living high at $2320 \mathrm{~m}$ asl does not produce adverse effects on muscular function in elite swimmers, even if the training is not solely focus on improving force and power [73]. In this study, the same group of swimmers were compared before and after 3 weeks of training at sea level at moderate altitude. Evaluations were separated by a 1-year period, although the intervention period, training targets, and relative loads were maintained. No changes in swimming start times were observed after the altitude period, while an impairment was registered at sea level. No differences between conditions were obtained during the loaded SJ performance despite a slight improvement in peak velocity after both training periods (Table 3).

The approximate $22.9 \%$ difference in air density at moderate altitude $(\sim 3 \%$ reduction for each $305 \mathrm{~m}$ rise; [5]) could contribute to making the movement faster than at normal altitudes. More studies are needed in order to analyze if an interaction exists between air pressure and composition, as well as examining the effects of longer training periods in these conditions. Researchers have identified [14, 74-76] and ruled out [77-79] differences in the physiological responses to exercise when comparing $\mathrm{NH}$ and $\mathrm{HH}$. Millet et al. [76] reported power output improvements of 4.0 and $4.2 \%$ for elite and non-elite athletes, respectively, in conditions of $\mathrm{HH}$ vs 0.6 and $1.4 \%$ for $\mathrm{NH}$. Bonetti and Hopkins [80] described increased ventilatory responses, changes in fluid balance, and nitric oxide metabolism, along with changes in the severity of acute mountain sickness and altered performance for $\mathrm{HH}$ compared to those for $\mathrm{NH}$. However, these studies assessed the effects of chronic hypoxia, and no explanation has yet been offered for muscle power differences related to acute and longer periods spent at altitude.

\section{Inconsistencies Among Studies Conducted into the Effect of Hypoxia on Muscle Power Resistance Training}

The influences of whatever type of hypoxia on musclespecific performance in sport have not been thoroughly examined. While its causes remain unclear, we have identified a mechanism which could positively affect the performance of and training for isolated explosive actions at terrestrial moderate altitude. Muscle force or power development in hypoxic conditions is not a variable that is commonly assessed in scientific publications. From the nine studies included in this part of the review, five analyze the effect of acute exposure and contain measurements at different severities $\left(\mathrm{FiO}_{2}\right.$ from 16 to $13 \%$ ) and for different types of hypoxia (systemic vs terrestrial altitude). They examine the effect of various types of exercises (jumps, squat, deadlift, or bench press) and include subjects of differing sporting ability (elite vs non-trained). The remaining studies are conducted at real or simulated hypobaric hypoxia of around 2400 asl and analyze the effect of different types of resistance training on free or loaded jumps (SJ and CMJ). The presence of a control group, group size, sex distribution in groups, training level, or description of the training and assessment process are some of the differences observed among the abovementioned longitudinal studies. Finally, training orientation is also different among the studies. While Âlvarez-Herms et al. [71] described resistance endurance training to improve anaerobic power during multiple jumps, García-Ramos et al. [42, 72] did not implement the study with specific tasks. The authors did however indicate that six of the ten resistance training exercises were oriented to strength-power training in the legs $[42,72]$. Additionally, the concurrent strength and endurance training used in the García-Ramos et al. [73] study corroborate that an excessively oriented training aiming to improve endurance capacity attenuates strength training responses [81], even after an altitude training camp of 3 weeks [73, 82].

Controlled and power-oriented resistance training studies are clearly needed to analyze the effect of intermittent or sustained altitude exposure on power training.

\section{Conclusions}

Current evidences suggest potentially promising applications of hypoxia for muscle hypertrophy and power training. Nevertheless, there is still insufficient data on which to base training programs. To help design altitude training protocols, data from more specific controlled studies are needed.

\section{In Hypertrophy}

The evidence for greater muscle strength gains and structural physiological changes in response to resistance training under conditions of hypoxia is not conclusive. This is because although the balance of results tends to favor training in hypoxia, only one study revealed significant differences in performance between resistance training in normoxia and training in hypoxia.

Currently, the definitive mechanisms that may augment muscular responses to hypertrophy resistance training under hypoxic conditions are not yet fully understood. However, despite a need for further research, it may be reasonably suggested that (1) metabolite build-up during low-intensity $(\leq 30 \% 1 \mathrm{RM})$ resistance exercise may be intensified in hypoxia; (2) greater and faster changes may occur in hypoxia when multiple sets of 6-12 repetitions at moderate load ( $\geq 65 \% 1 \mathrm{RM})$ are performed; and (3) the recommended simulated hypoxia level for all training modalities is moderate $\left(13-16 \% \mathrm{FiO}_{2}\right)$. 
Preliminary studies seem to indicate that hypertrophyoriented training conducted under conditions of intermittent hypoxia could promote more favorable physiological and functional changes than under chronic exposure. Terrestrial or simulated living low-training high strategies seem to benefit anabolic responses.

Issues related to nutrition and hydration, as well as the adjustment of the training load due to the possible influence of an ascent in altitude on the 1RM estimation (to avoid reducing muscle stimulus and muscle mass), should also be taken into account when spending long periods at altitude.

\section{In Muscle Power Development}

Ascent to altitude leads to velocity and power improvements although the mechanisms that promote the benefit of this type of hypoxia with respect to the $\mathrm{NH}$ still require clarification. Athletes should not be excessively concerned about the deterioration of muscular function when they take part of a 2-3-week training period at moderate altitude, even if the training is not strongly oriented to force and power development.

The following points should be considered in an altitude power-oriented training program: (1) loads used for power training under normal conditions should not be literally translated to training programs performed at higher altitudes. This is especially relevant because of the importance of locating and assessing the optimal muscular load for power training programs; (2) load adjustments during resistance training sessions at terrestrial altitude (according to the altitude 1RM) avoid reducing the muscle stimulus and/or inter- and intramuscle coordination that commonly occurs after periods of altitude training; (3) F-V curves emerging from the different studies, despite involving different resistances, correspond to exercise volumes that do not induce local metabolic fatigue and could thus compromise muscle contractile properties. Protocols with inter-repetition or intra-set rest periods (cluster training) might therefore be more suitable for hypoxic resistance training focus on this topic.

Unlike simulated hypoxia, terrestrial altitude conditions seem to improve the ability to perform high-speed actions with moderate loads. Thus, training under these conditions could serve to improve velocity and technical skills in power-related sports.

\section{Funding}

This review was supported by the Spanish Ministry of Science and Innovation (DEP2012-35774; DEP2015-64350-P [MINECO/FEDER]), Ministry of Education, Culture and Sport.

\section{Authors' Contributions}

$\mathrm{BF}$ conceived, designed, drafted, and revised the manuscript; contacted the authors; and interpreted the findings; BF, AGR, AMA, and PP executed the review, interpreted the findings, and contributed to the writing of the paper, tables, and figures. All authors read and approved the final manuscript.

\section{Competing interests}

Belén Feriche, Antonio Jesús Morales-Artacho, Amador García-Ramos and Paulino Padial declare that they have no competing interests.

Consent for Publication

Not applicable

Ethics Approval and Consent to Participate

Not applicable

Received: 20 September 2016 Accepted: 23 February 2017

Published online: 17 March 2017

References

1. Hamlin MJ, Hopkins WG, Hollings SC. Effects of altitude on performance of elite track-and-field athletes. Int J Sports Physiol Perform. 2015;10:881-7.

2. Hollings SC, Hopkins WG, Hume PA. Environmental and venue-related factors affecting the performance of elite male track athletes. Eur J Sport Sci. 2012;2:201-6.

3. McLean BD, Buttifant D, Gore CJ, White K, Liess C, Kemp J. Physiological and performance responses to a preseason altitude-training camp in elite teamsport athletes. Int J Sports Physiol Perform. 2013;8:391-9.

4. Saunders PU, Ahlgrim C, Vallance B, Green DJ, Robertson EY, Clark SA, et al. An attempt to quantify the placebo effect from a three-week simulated altitude training camp in elite race walkers. Int J Sports Physiol Perform. 2010:5:521-34.

5. Levine BD, Stray-Gundersen J, Mehta RD. Effect of altitude on football performance. Scand J Med Sci Sports. 2008;18:76-84.

6. Faiss R, Girard O, Millet GP. Advancing hypoxic training in team sports: from intermittent hypoxic training to repeated sprint training in hypoxia. $\mathrm{Br}$ J Sports Med. 2013:47:i45-50.

7. Brocherie F, Girard O, Faiss R, Millet GP. High-intensity intermittent training in hypoxia: a double-blinded, placebo-controlled field study in youth football players. J Strength Cond Res. 2015;29:226-37.

8. Kraemer WJ, Ratamess NA. Fundamentals of resistance training: progression and exercise prescription. Med Sci Sports Exerc. 2004;36:674-88.

9. Schoenfeld BJ. The mechanisms of muscle hypertrophy and their application to resistance training. J Strength Cond Res. 2010;24:2857-72.

10. Cormie P, McGuigan MR, Newton RU. Developing maximal neuromuscular power: part 2 training considerations for improving maximal power production. Sports Med. 2011;41:125-46.

11. Millet GP, Faiss R, Brocherie F, Girard O. Hypoxic training and team sports: a challenge to traditional methods? Br J Sports Med. 2013;47:i6-7.

12. Wilber RL. Application of altitude/hypoxic training by elite athletes. Med Sci Sports Exerc. 2007;39:1610-24.

13. Scott BR, Slattery KM, Dascombe BJ. Intermittent hypoxic resistance training: Is metabolic stress the key moderator? Med Hypotheses. 2015;84:145-9.

14. Friedmann B, Kinscherf R, Borisch S, Richter G, Bartsch P, Billeter R. Effects of low-resistance/high-repetition strength training in hypoxia on muscle structure and gene expression. Pflugers Arch. 2003;446:742-51.

15. Ho JY, Kuo TY, Liu KL, Dong XY, Tung K. Combining normobaric hypoxia with short-term resistance training has no additive beneficial effect on muscular performance and body composition. J Strength Cond Res. 2014:28:935-41.

16. Kon M, Ohiwa N, Honda A, Matsubayashi T, Ikeda T, Akimoto T, et al. Effects of systemic hypoxia on human muscular adaptations to resistance exercise training. Physiol Rep. 2014;2:1-13.

17. Kurobe K, Huang Z, Nishiwaki M, Yamamoto M, Kanehisa H, Ogita F. Effects of resistance training under hypoxic conditions on muscle hypertrophy and strength. Clin Physiol Funct Imaging. 2015;35:197-202.

18. Abe T, Yasuda T, Midorikawa T, Sato Y, Kearns CF, Inoue K, et al. Skeletal muscle size and circulating IGF-1 are increased after two weeks of twice daily 'KAATSU' resistance training. Int J Kaatsu Training Res. 2005;1:6-12.

19. Karabulut M, Abe T, Sato Y, Bemben M. Overview of neuromuscular adaptations of skeletal muscle to KAATSU Training. Int J Kaatsu Training Res. 2007;3:1-9.

20. Madarame H, Neya M, Ochi E, Nakazato K, Sato Y, Ishii N. Cross-transfer effects of resistance training with blood flow restriction. Med Sci Sports Exerc. 2008;40:258-63. 
21. Manimmanakorn A, Hamlin MJ, Ross JJ, Taylor R, Manimmanakorn N. Effects of low-load resistance training combined with blood flow restriction or hypoxia on muscle function and performance in netball athletes. J Sci Med Sport. 2013;16:337-42.

22. Manimmanakorn A, Manimmanakorn N, Taylor R, Draper N, Billaut F, Shearman JP, et al. Effects of resistance training combined with vascular occlusion or hypoxia on neuromuscular function in athletes. Eur J Appl Physiol. 2013;113:1767-74.

23. Yasuda T, Fujita S, Ogasawara R, Sato Y, Abe T. Effects of low-intensity bench press training with restricted arm muscle blood flow on chest muscle hypertrophy: a pilot study. Clin Physiol Funct Imaging. 2010;30:338-43.

24. Scott BR, Slattery KM, Dascombe BJ. Intermittent hypoxic resistance training: does it provide added benefit? Front Physiol. 2014;5:397.

25. Fry CS, Glynn EL, Drummond MJ, Timmerman KL, Fujita S, Abe T, et al. Blood flow restriction exercise stimulates mTORC1 signaling and muscle protein synthesis in older men. J Appl Physiol. 2010;108:1199-209.

26. Loenneke JP, Wilson GJ, Wilson JM. A mechanistic approach to blood flow occlusion. Int J Sports Med. 2010;31:1-4.

27. Schoenfeld BJ. Potential mechanisms for a role of metabolic stress in hypertrophic adaptations to resistance training. Sports Med. 2013;43:179-94.

28. Kon M, Ikeda T, Homma T, Suzuki Y. Effects of low-intensity resistance exercise under acute systemic hypoxia on hormonal responses. J Strength Cond Res. 2012;26:611-7.

29. Scott BR, Slattery KM, Sculley DV, Dascombe BJ. Hypoxia and resistance exercise: a comparison of localized and systemic methods. Sports Med. 2014;44:1037-54.

30. Kon M, Ikeda T, Homma T, Akimoto T, Suzuki Y, Kawahara T. Effects of acute hypoxia on metabolic and hormonal responses to resistance exercise. Med Sci Sports Exerc. 2010;42:1279-85.

31. Nishimura A, Sugita M, Kato K, Fukuda A, Sudo A, Uchida A. Hypoxia increases muscle hypertrophy induced by resistance training. Int J Sports Physiol Perform. 2010;5:497-508.

32. Hahn AG, Gore CJ. The effect of altitude on cycling performance: a challenge to traditional concepts. Sports Med. 2001;31:533-57.

33. Peronnet $F$, Thibault $G$, Cousineau DL. A theoretical analysis of the effect of altitude on running performance. J Appl Physiol. 1991;70:399-404.

34. Balsom PD, Gaitanos GC, Ekblom B, Sjödin B. Reduced oxygen availability during high intensity intermittent exercise impairs performance. Acta Physiol Scand. 1994;152:279-85

35. Billaut F, Gore CJ, Aughey RJ. Enhancing team-sport athlete performance: is altitude training relevant? Sports Med. 2012;42:751-67.

36. Chapman RF, Laymon Stickford AS, Lundby C, Levine BD. Timing of return from altitude training for optimal sea level performance. J Appl Physiol. 2014;116:837-43.

37. Delliaux S, Jammes Y. Effects of hypoxia on muscle response to tendon vibration in humans. Muscle Nerve. 2006;34:754-61.

38. Tomazin K, García-Ramos A, Feriche B, Strojnik V, Stirn I. Acute exposure to moderate altitude increased spinal excitability. 21st Annual Congress of European College of Sport Science. 2016.

39. Melissa L, MacDougall JD, Tarnopolsky MA, Cipriano N, Green HJ. Skeletal muscle adaptations to training under normobaric hypoxic versus normoxic conditions. Med Sci Sports Exerc. 1997;29:238-43.

40. Feriche B, García-Ramos A, Calderón-Soto C, Drobnic F, Bonitch-Góngora JG, Galilea PA, et al. Effect of acute exposure to moderate altitude on muscle power: hypobaric hypoxia vs. normobaric hypoxia. PLoS One. 2014;9:e114072.

41. Chirosa I, Feriche B, Calderón C, Martínez M, Braga R, Padial P. Ascent to moderate altitude improves force production. Arch Med Dep. 2006;23:101-8.

42. García-Ramos A, Argüelles J, de la Fuente B, Padial P, Bonitch J, Calderón C, et al. Performance of muscular power profile after a training camp at moderate natural altitude in young swimmers. J Strength Cond Res. 2014;28:85.

43. García-Ramos A, Štirn I, Padial P, Argüelles-Cienfuegos J, De la Fuente B, Strojnik $V$, et al. The maximal mechanical capabilities of leg extensors muscles to generate velocity and power improve at altitude. J Strength Cond Res. 2016. doi:10.1519/JSC.0000000000001592.

44. Scott BR, Slattery KM, Sculley DV, Hodson JA, Dascombe BJ. Physical performance during high-intensity resistance exercise in normoxic and hypoxic conditions. J Strength Cond Res. 2015;29:807-15.

45. Mizuno M, Savard GK, Areskog NH, Lundby C, Saltin B. Skeletal muscle adaptations to prolonged exposure to extreme altitude: a role of physical activity? High Alt Med Biol. 2008:9:311-7.

46. Narici MV, Kayser B. Hypertrophic response of human skeletal muscle to strength training in hypoxia and normoxia. Eur J Appl Physiol Occup Physiol. 1995;70:213-9.
47. Brouns F. Nutritional aspects of health and performance at lowland and altitude. Int J Sports Med. 1992;13:S100-6.

48. Chaudhary P, Suryakumar G, Prasad R, Singh SN, Ali S, llavazhagan G. Chronic hypobaric hypoxia mediated skeletal muscle atrophy: role of ubiquitinproteasome pathway and calpains. Mol Cell Biochem. 2012;364:101-13.

49. Koumenis C, Wouters BG. 'Translating' tumor hypoxia: unfolded protein response (UPR)-dependent and UPR-independent pathways. Mol Cancer Res. 2006;4:423-36.

50. Etheridge T, Atherton PJ, Wilkinson D, Selby A, Rankin D, Webborn N, et al Effects of hypoxia on muscle protein synthesis and anabolic signaling at rest and in response to acute resistance exercise. Am J Physiol Endocrinol Metab. 2011;301:E697-702.

51. Ho JY, Huang TY, Chien YC, Chen YC, Liu SY. Effects of acute exposure to mild simulated hypoxia on hormonal responses to low-intensity resistance exercise in untrained men. Res Sports Med. 2014:22:240-52.

52. West DW, Phillips SM. Anabolic processes in human skeletal muscle: restoring the identities of growth hormone and testosterone. Phys Sportsmed. 2010;38:97-104.

53. Izquierdo M, González-Badillo JJ, Häkkinen K, Ibáñez J, Kraemer WJ, Altadill $A$, et al. Effect of loading on unintentional lifting velocity declines during single sets of repetitions to failure during upper and lower extremity muscle actions. Int J Sports Med. 2006;27:718-24.

54. Sánchez-Medina L, González-Badillo JJ. Velocity loss as an indicator of neuromuscular fatigue during resistance training. Med Sci Sports Exerc. 2011:43:1725-34.

55. Fry AC. The role of resistance exercise intensity on muscle fibre adaptations. Sports Med. 2004:34:663-79.

56. Moritani T, DeVries HA. Neural factors versus hypertrophy in the time course of muscle strength gain. Am J Phys Med. 1979;58:115-30.

57. Calderón C, Rodríguez F, Iglesias X, Feriche B, Pérez I, Trujiens MJ, et al. Acute mountain sickness during a natural altitude training camp in elite swimmers. 16th Annual Congress of European College of Sport Science. 2011.

58. Charlot K, Pichon A, Richalet JP, Chapelot D. Effects of a high-carbohydrate versus high-protein meal on acute responses to hypoxia at rest and exercise. Eur J Appl Physiol. 2013;113:691-702.

59. Trujiens $M$, Rodríguez F. Altitude and hypoxic training in swimming. In: Seifert L, Chollet D, Mujika I, editors. World book of swimming: from science to performance. 2010

60. Smith KJ, Billaut F. Influence of cerebral and muscle oxygenation on repeated-sprint ability. Eur J Appl Physiol. 2010;109:989-99.

61. Amann M, Eldridge MW, Lovering AT, Stickland MK, Pegelow DF, Dempsey JA. Arterial oxygenation influences central motor output and exercise performance via effects on peripheral locomotor muscle fatigue in humans. J Physiol. 2006;575:937-52.

62. Millet GY, Aubert D, Favier FB, Busso T, Benoît H. Effect of acute hypoxia on central fatigue during repeated isometric leg contractions. Scand J Med Sci Sports. 2009;19:695-702

63. Perrey S, Rupp T. Altitude-induced changes in muscle contractile properties. High Alt Med Biol. 2009;10:175-82.

64. Ferretti $\mathrm{G}$, Hauser $\mathrm{H}$, di Prampero PE. Maximal muscular power before and after exposure to chronic hypoxia. Int J Sports Med. 1990;11:S31-4.

65. Samozino P, Rejc E, Di Prampero PE, Belli A, Morin JB. Optimal force-velocity profile in ballistic movements-altius: citius or fortius? Med Sci Sports Exerc. 2012:44:313-22.

66. Jidovtseff B, Harris NK, Crielaard JM, Cronin JB. Using the load-velocity relationship for 1RM prediction. J Strength Cond Res. 2011;25:267-70.

67. Bazuelo-Ruiz B, Padial P, García-Ramos A, Morales-Artacho AJ, Miranda MT, Feriche B. Predicting maximal dynamic strength from the load-velocity relationship in squat exercise. J Strength Cond Res. 2015;29:1999-2005.

68. González-Badillo JJ, Sánchez-Medina L. Movement velocity as a measure of loading intensity in resistance training. Int J Sports Med. 2010;31:347-52.

69. Amann M, Romer LM, Subudhi A, Pegelow DF, Dempsey JA. Severity of arterial hypoxaemia affects the relative contributions of peripheral muscle fatigue to exercise performance in healthy humans. J Physiol. 2007;581:389-403.

70. Henslin Harris KB, Foster C, De Koning JJ, Dodge C, Wright GA, Porcari JP. Rapidity of response to hypoxic conditions during exercise. Int J Sports Physiol Perform. 2013;8:330-5.

71. Álvarez-Herms J, Julià-Sánchez S, Corbi F, Pagès T, Viscor G. Anaerobic performance after endurance strength training in hypobaric environment. Sci Sports. 2014;29:311-8. 
72. Garcia-Ramos A, Padial P, Fuente B, Arguelles-Cienfuegos J, BonitchGongora J, Feriche B. Relationship between vertical jump height and swimming start performance before and after an altitude training camp. J Strength Cond Res. 2016:30:1638-45.

73. García-Ramos A, Štirn I, Padial P, Argüelles-Cienfuegos J, De la Fuente B,

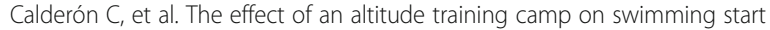
time and loaded squat jump performance. PLoS One. 2016;11:e0160401.

74. Fulco CS, Friedlander AL, Muza SR, Rock PB, Robinson S, Lammi E, et al. Energy intake deficit and physical performance at altitude. Aviat Space Environ Med. 2002;73:758-65.

75. Saugy JJ, Schmitt L, Cejuela R, Faiss R, Hauser A, Wehrlin JP, et al. Comparison of 'live high-train low' in normobaric versus hypobaric hypoxia. PLoS One. 2014;9:e114418.

76. Millet GP, Faiss R, Pialoux V. Point: hypobaric hypoxia induces/does not induce different responses from normobaric hypoxia. J Appl Physiol. 2012:112:1783-4.

77. Geiser J, Vogt M, Billeter R, Zuleger C, Belforti F, Hoppeler H. Training highliving low: changes of aerobic performance and muscle structure with training at simulated altitude. Int J Sports Med. 2001;22:579-85.

78. Siebenmann C, Robach P, Jacobs RA, Rasmussen P, Nordsborg N, Diaz V, et al. 'Live high-train low' using normobaric hypoxia: a double-blinded, placebo-controlled study. J Appl Physiol. 2012;112:106-17.

79. Robach P, Schmitt L, Brugniaux JV, Roels B, Millet G, Hellard P, et al. Living high-training low: effect on erythropoiesis and aerobic performance in highly-trained swimmers. Eur J Appl Physiol. 2006;96:423-33.

80. Bonetti DL, Hopkins WG. Sea-level exercise performance following adaptation to hypoxia: a meta-analysis. Sports Med. 2009;39:107-27.

81. Rønnestad BR, Hansen EA, Raastad T. High volume of endurance training impairs adaptations to 12 weeks of strength training in well-trained endurance athletes. Eur J Appl Physiol. 2012;112:1457-66.

82. Strojnik V, Tomazin K, Stirn I, Strumbelj B, Garcia-Ramos A, Padial P, et al. Effects of altitude concurrent strength and endurance training on neuromuscular function. 21st Annual Congress of European College of Sport Science. 2016

\section{Submit your manuscript to a SpringerOpen ${ }^{\circ}$ journal and benefit from:}

- Convenient online submission

- Rigorous peer review

- Immediate publication on acceptance

- Open access: articles freely available online

- High visibility within the field

- Retaining the copyright to your article 